[20] R. J. Koch, Ordered semigroups in partially ordered semigroups, Pacific Journ. of Math 10 (1960), pp. 1333-1338.

[21] - On monothetic semigroups, Proc. A.M.S. 8 (1957), pp. 397-401.

[22] - Remarks on primitive idempotents in compact semigroups with zero, Proc. A.M.S. 5 (1954), pp. 828.833.

[23] P. S. Mostert and A. L. Shields, One parameter semigroups in a semigroup, Trans. A.M.S. 96 (1960), pp. 510-517.

[24] - One the structure of semigroups on a compact manifold with boundary, Annals of Math. 65 (1957), pp. 117-143.

[25] D. Montgomery and L. Zippin, Topological transformation groups, Interscience.

[26] D. Rees, On semigroups, Proc. Cambridge Phil. Soc. 36 (1940), pp. 387-400.

[27] - Note on semigroups, Proc. Cambridge Phil. Soc., 37 (1941), pp. 434-435.

[28] N. Steenrod, The topology of fibre bundles, Princeton U. Press.

[29] G. Thierrin, Contribution à la théorie des équivalences dans les demi-groupes, Thèse Paris (1954), Bull. Soc. Math. France 83 (1955), pp. 103-159.

[30] - Sur la caractérisation des groupes par leurs équivalenoes réguliers, C. R. Acad. Sci. Paris 238 (1954), pp. 1954-1956.

[31] - Sur la structure des demi-groupes, Algèr Mathématique, Tome III, no. 2 (1956), pp. 161-171.

[32] A. D. Wallace, The structure of topological semigroups, Bull. Amer. Math. Soc. 61 (1955), pp. 95-112.

[33] - Cohomology, dimension, and semigroups, Summa Brasil Math., 3 Fasc. 5, August 1953.

[34] - The Rees Suschkewitsch structure theorem for compact simple semigroups, Proc. Nat. Acad. Sci. 42 (1956), pp. 430-432.

[35] - The position of $O-$-sets in semigroups, Proc. A.M.S. 6 (1955), pp. 639-642.

[36] G. T. Whyburn, Analytio topology, Amer. Math. Soc. Coll. Pub., Vol. 28 (1951).

THE UNIVERSITY OF GEORGIA

and THE PENNSYLVANIA STATE UNIVERSITY

Reģu par la Rédaction le 13. 11. 1961

\section{Pointwise periodic groups}

by

K. H. Hofmann* (Tübingen) and F. B. Wright** (New Orleans, La.)

1. Introduction. Let $A$ be any Boolean ring, and let $G$ be a group of automorphisms of $A$; for an element $a \in A$ and an element $g \in G$, we denote the image of $a$ under $g$ by $a^{g}$. The set $a^{G}=\{a: g \in G\}$ is called the orbit of $a$ (under $G$ ). The group $G$ is said to be pointwise periodic (on $A$ ) if every orbit is a finite set, and a pointwise periodic group is said to be periodic (on $A$ ) if there is an upper bound to the size of the orbits. (A group, all of whose elements have finite order, is sometimes called periodic. We shall call such groups torsion groups.) By the duality theory of M. H. Stone [6], any Boolean ring is isomorphic to the Boolean field of open compact sets in some locally compact, totally disconnected, Hausdorff space $X$. Following Stone, we shall call such spaces Boolean spaces, and, following Halmos, we shall the space $X$ associated with a Boolean ring the dual space of the ring. Stone has shown that there is a natural isomorphism between the group of all automorphisms of a Boolean ring and the group of all homeomorphisms of its dual space. Consequently, for any group $G$ of automorphisms of $A$ there is an isomorphic group $\Gamma$ of homeomorphisms of $X$. The notions of pointwise periodicity and periodicity for $\Gamma$ have an obvious meaning.

The special case of a cyclic group has received some attention. More precisely, if $g$ is an automorphism of $A$, and if $\gamma$ is the dual homeomorphism of $X$, then the following is known:

(a) The automorphism $g$ is periodic if and only if $\gamma$ is periodic; this is an immediate consequence of the isomorphism given by Stone.

(b) If $\gamma$ is periodic, then, trivially, it is pointwise periodic.

(c) If $\gamma$ is pointwise periodic, then $g$ is pointwise periodic; this result is due to A. D. Wallace [7], extending a theorem of Hall and Schweigert [2].

The converses of (b) and (c) are not, in general, valid. Counter-examples will be found below; the failure of the converse of (c) seems not to have been noticed.

* Research supported by the National Science Foundation;

** Research Fellow of the Alfred P. Sloan Foundation. 
If $G$ is an arbitrary group of automorphisms, with dual group $\Gamma$ of homeomorphisms, then the situation is more complex. The following assertions are then valid:

(i) If $G$ is periodic, then $\Gamma$ is periodic,

(ii) If $\Gamma$ is periodic, then, trivially, it is pointwise periodic.

Statement (i) will be proved below, and an example will be adduced to show the failure of the converse. The analogue of (c) can be proved only under an additional hypothesis:

(iii) If $\Gamma$ is pointwise periodic and if $X$ has a Hausdorff orbit space under $\Gamma$, then $G$ is pointwise periodic.

This will be proved below in a manner somewhat similar to Wallace's proof in the cyclic case. Examples show that the assumption of a Hausdorff orbit space cannot be dropped. An equivalent formulation, in dynamical terms, is the following:

(iii') If $\Gamma$ is pointwise periodic and locally weakly almost periodic, then $G$ is pointwise periodic.

The converse of each of these fails, even if the group is a countable, abelian, torsion group in which every element has order 2.

It thus appears that the weakest hypothesis which can, in general, be imposed in this situation is that the automorphism group $G$ be pointwise periodic on the Boolean algebra $A$. It is somewhat surprising that, if $A$ is assumed to be a Boolean $\sigma$-algebra, this hypothesis is equivalent to the strongest of all possible "periodicity" assumptions about $G$, namely, that $G$ is a finite group. In topological terms, this is the assertion that a pointwise periodic and locally weakly almost periodic group of homeomorphisms of a Boolean $\sigma$-space is necessarily a finite group. (In particular, these apply to complete Boolean algebras and extremally disconnected Boolean spaces.) As might be expected, the proof of this is somewhat long, and is quite combinatorial in character.

A classic result of Montgomery [5] asserts that a pointwise periodic homeomorphism of a connected locally Euclidean space is necessarily periodic. Our results may be regarded as a fairly complete analysis of what can happen at the other end of the topological spectrum; that is, when the space is totally disconnected. In particular, Theorem VII may be considered as the analogue of Montgomery's theorem.

2. Preliminaries. If $A$ is a Boolean ring, we shall write $X=A^{*}$ to denote that the (locally compact) Boolean space $X$ is the dual space of $A$. Similarly, we shall, for any locally compact Boolean space $X$, write $A=X^{*}$ to denote the Boolean algebra of all compact open sets of $X$. If $g$ is an automorphism of $A$, we denote the corresponding homeomorphism $\gamma$ by writing $\gamma=g^{*}$ and $g=\gamma^{*}$. If $G$ is a group of automorphisms of $A$, and if $\Gamma$ is the corresponding group of homeomorphisms of $X$, we write $\Gamma=G^{*}$ and $G=\Gamma^{*}$. The compact open set $U$ which corresponds to an element $a$ of $A$ will be denoted as $U=a^{*}$. The image of an element $a \in A$ under $g \in G$ will be denoted by $a^{g}$; the image of a point $x \in X$ under $\gamma \in \Gamma$ will be written $\gamma x$. If $a \in A, g \in G, U=a^{*}, \gamma=g^{*}$, then $\left(a^{g}\right)^{*}=\gamma^{-1} U$.

If $G$ is a group of automorphisms of $A$, the orbit of an element $a \in A$ is the set $a^{G}=\left\{a^{g}: g \in G\right\}$. The cardinal number of the orbit of $a$ is called the order of $a$, and will be denoted by $o(a)$. The group $G$ is said to be pointwise periodic if $o(a)$ is finite for every $a \in A$, and is said to be periodic if it is pointwise periodic and if the orders are bounded. An element $a \in A$ is called an invariant element if $o(a)=1$; that is, if $a^{o}=a$ for all $g \in G$. For each element $a \in A$, we let $G_{a}=\left\{g \in G: a^{g}=a\right\}$, and we call this subgroup the fix subgroup of $G$. The order of $a$ is then equal to the index $\left(G: G_{a}\right)$ of $G_{a}$ in $G$.

The same terminology and notation will be applied to a group of homeomorphisms of $X$, with one exception: A point $x \in X$ will be called a fixed point if $\gamma x=x$ for all $\gamma \in \Gamma$.

The concept embodied in the first definition will play a fundamental role throughout this paper.

2.1. Definition. If $G$ is a group of automorphisms of a Boolean algebra $A$, an element $a \in A$ is called an orbital atom if, for any $g \in G$, $a^{g} \wedge a \neq 0$ implies $a^{g}=a$.

Orbital atoms do not, in general, exist. For pointwise periodic groups, however, they are abundantly present.

2.2. Proposition. If $G$ is a pointwise periodic automorphism group of $A$, then every non-zero element a in $A$ can be written as a disjoint union of a finite set of non-zero orbital atoms.

Proof. The set $a^{G}$ is finite, and generates a finite subring of $A$. This subring is completely atomic and its atoms are permuted by $G$. The atoms of this ring are therefore orbital atoms, and every element is the disjoint union of such orbital atoms.

2.3. Proposition. (i) Any invariant element is an orbital atom.

(ii) The intersection of two orbital atoms is an orbital atom.

(iii) If $a \geqslant b \neq 0$, and if $a$ is an orbital atom, then $G_{b} \subset G_{a}$; in particular, if $G$ is pointwise periodic, then $o(a)$ divides $o(b)$.

Proof. (i) and (ii) are trivial; we prove (iii) as a sample of the calculations used repeatedly with orbital atoms. If $b^{g}=b$, then $a^{g} \wedge a \geqslant b^{g} \wedge b$ $=b \neq 0$, so that $a^{g}=a$.

2.4. DEFINITION. If $G$ is a pointwise periodic group of automorphisms of $A$, we define, for any element $a \in A$, the element $\bar{a}=\sup a^{\alpha}$. 
We note explicitly that this supremum exists, since the set $a^{G}$ is finite when $G$ is pointwise periodic. (The meaning of the mapping $a \rightarrow \bar{a}$ when $G$ is not pointwise periodic is discussed in [8].)

2.5. Propostiton. Let $G$ be pointwise periodic.

(i) An element $a$ is invariant if and only if $a=\bar{a}$.

(ii) If $a$ is invariant, then $\overline{a \wedge b}=\bar{a} \wedge \bar{b}$ for any $b \in A$.

(iii) If $E \subset A$ is a subset of $A$ such that inf $E=0$ exists in $A$, then for any element $a \epsilon A$, we have $\overline{a \wedge c}=\bar{a} \wedge \bar{c}$, provided that $E$ consists of invariant elements.

Proof. (i) is obvious, (ii) follows from the distributive law, and (iii) follows from (ii).

3. Pointwise periodicity. This section is devoted to establishing statements (i) and (iii) in the introduction.

3.1. Proposition. Let $G$ be a pointwise periodic group of automorphisms of a Boolean ring $A$, and suppose that the order of any orbital atom of $A$ is $<q$, for some natural number $q$. Then the dual group $\Gamma=G^{*}$ is a periodic group of homeomorphisms of $X=A^{*}$, and the order of any point in $X$ is $<q$.

Proof. Any compact open set in $X$ is the disjoint union of a finite collection of orbital atoms, by 2.2. (A compact open set $U \subset X$ is called an orbital atom for $\Gamma$ if $U=a^{*}$, where $a$ is an orbital atom in $A$.) Hence the orbital atoms in $X$ form a basis for the topology of $X$. Let $x$ be a point which has at least $q$ different points $x=\gamma_{1} x, \gamma_{2} x, \ldots, \gamma_{q} x$ in its orbit. Then all of the points $\gamma_{j}^{-1} \gamma_{i} x, i, j=1, \ldots, q$, are different from $x$ provided $i \neq j$. Hence we can find a sufficiently small neighborhood $U_{i j}$ of $x$, which is an orbital atom, such that $U_{i j} \cap \gamma_{j}^{-1} \gamma_{i} U_{i j}=\emptyset, i, j=1, \ldots, q, i \neq j$. Then the intersection $U$ of all the $U_{i j}$ is an orbital atom and a neighborhood of $x$ such that $\gamma_{i} U \cap \gamma_{i} U=\varnothing$ for $i, j=1, \ldots, q, i \neq j$. But this contradicts the fact that $U$ does not admit $q$ disjoint translates. This contradiction proves the proposition.

As an immediate corollary, we have the following result:

THeOREM I. If $G$ is a periodic group of automorphisms of a Boolean ring $A$, then its dual group $\Gamma=G^{*}$ is a periodic group of homeomorphisms of the dual space $X=A^{*}$.

In the fifth section of the paper, we give an example to show that the converse does not hold, in general. The example will, in fact, show that $\Gamma$ may be periodic on $X$ and $G$ pointwise periodic so that the orders of orbital atoms are bounded, while $G$ is still not periodic (see 5.4.).

3.2. Lemma. Let $X$ be any topological space, let $x \in X$, and let $\mathcal{U}$ be a family of compact neighborhoods of $x$ such that $\cap\{U: U \in \mathscr{\ell}\}=\{x\}$. Then $\mathcal{U}$ is a neighborhood basis for $x$.
Proof. Let $\nabla$ be any open neighborhood of $x$. If no element $W$ of $\mathcal{U}$ is contained in $\nabla$, then the filter basis $\{W \backslash \nabla: W \in \mathcal{U}\}$ of compact sets has a non-void intersection which is in the complement of $\nabla$, and therefore does not contain $x$. This is a contradiction.

3.3. Lemma. Let $X$ be any topological space, let $Y$ be any topological $T_{1}$-space and let $\pi$ be a continuous mapping of $X$ onto $Y$. Let $x$ be a point in $X$ for which the following conditions are satisfied:

(i) $\pi(x)$ has a basis $\mathcal{U}$ of neighborhoods such that $\pi^{-1} U$ is compact for each $U \in \mathcal{U}$

(ii) $\pi^{-1} \pi(x)=\{x\}$.

Then the family $\left\{\pi^{-1} U: U \in \mathcal{U}\right\}$ is a neighborhood basis for $x$.

Proof. Because of 3.2 , it suffices to show that $\bigcap \pi^{-1} U=\{x\}$. Suppose $y \neq x$. By condition (ii), $\pi(y) \neq \pi(x)$. Since $Y$ is a $T_{1}$-space, there is a $U \in \mathcal{U}$ such that $\pi(y) \notin U$. Then $y \notin \pi^{-1} U$.

3.4. LEMcra. Let $X$ be a locally compact Boolean space, and let $\Gamma$ be a pointwise periodic group of homeomorphisms of $X$. Suppose that, for each $x \in X$, there is a neighborhood basis of compact open sets $U$ having the property that $\gamma \in \Gamma_{x}$ implies $\gamma U=U$. Then the collection of all compact open sets which are orbital atoms form a basis for the topology of $X$. Moreover, if the orders of all points in $X$ divide a fixed natural number $q$, then the orders of all orbital atoms divide $q$.

Proof. Let $x \in X$, and let $U$ be an arbitrary neighborhood of $x$. Let $1=\gamma_{1}, \gamma_{2}, \ldots, \gamma_{n}$ be a system of representatives for the left cosets of $\Gamma_{x}$ in $\Gamma$. Then $\gamma_{i} x \neq x$ for all $i=2, \ldots, n$. Let $V$ be a neighborhood of $x$ contained in $U$ such that $\gamma_{i} \nabla \cap \nabla=\varnothing, i=2, \ldots, n$. Let $W$ be a compact open set in $V$ containing $x$, such that $\gamma \in \Gamma_{x}$ implies $\gamma W=W$. Then $W$ is an orbital atom. For if $\delta=\gamma_{i} \gamma, \gamma \in \Gamma_{x}$ is an arbitrary element of $I$ such that $\delta x \neq x$, then $i \neq 1$, since $\gamma x=x$. But now $\delta W=\gamma_{i} W$ does not intersect $W$ because $\gamma_{i} V$ does not intersect $V$. The remainder of the lemma is obvious since the intersection of two orbital atoms is an orbital atom, and since the order of an orbital atom divides the order of any of its points.

3.5. LEMMA. Let $\Gamma$ be a pointwise periodic group of homeomorphisms of a locally compact Boolean space such that the orbital atoms form a basis for the topology of $X$. Then any compact open set in $X$ is the union of a finite collection of orbital atoms.

Proof. Cover a compact open set with orbital atoms contained therein, and reduce to a finite covering.

Suppose now that $\Gamma$ is any group of homeomorphisms of a topological space $X$. The collection of orbits $\{\Gamma x: x \in X\}$ forms a partition of $X$ into equivalence classes. The quotient space $Y$ of $X$ modulo this equivalence 
relation forms a topological space called the orbit space of $X$ under $T$. This space is not, in general, even a $T_{1}$-space. In case $\Gamma$ is a pointwise periodic group on a $T_{1}$-space $X$, the orbits are finite, and hence the orbit space is a $T_{1}$-space. We shall give an example in section 5 below of a pointwise periodic $T$ operating on a compact Boolean space $X$ which has an orbit space which is not a Hausdorff space.

3.6. Definimion. A group $\Gamma$ of homeomorphisms of a topological space $X$ will be called a group with separated orbits if the orbit space of $X$ under $\Gamma$ is a Hausdorff space.

We shall consider conditions which guarantee that $T$ has separated orbits in the last section.

3.7. Lemara. Let $X$ be a compact Boolean space, let $T$ be a pointwise periodic group of homeomorphisms of $X$ with separated orbits, and let $x$ be a fixed point of $\Gamma$. Then $x$ has a neighborhood basis of compact open invariant sets.

Proof. Let $\pi$ be the projection mapping of $X$ onto the Hausdorff orbit space $Y$. If $U$ is any neighborhood of $\pi(x)$ in $Y$, then $\pi^{-1} U$ is a neighborhood of $x$ in $X$, and therefore contains a compact open neighborhood $V$ of $x$. The saturation $T V=\pi^{-1} \pi V$ is open. On the other hand, $\pi V$ is compact since $\pi$ is continuous and, since $Y$ is a Hausdorff space, $\pi V$ is closed in $Y$. Thus $\pi^{-1} \pi V$ is closed in $X$, and therefore is compact. Consequently, $U$ contains a compact open neighborhood $\pi V$ of $\pi(x)$ whose counter-image $\pi^{-1} \pi V$ is compact in $X$. The hypotheses of 3.3 are satisfied, and the collection $\pi^{-1} \pi V$, where $V$ is a compact open neighborhood of $x$, is the required basis of invariant neighborhoods.

For the case of a cyclic group, this result has been established by Wallace [7] for locally compact spaces. The same comment applies to the following:

3.8. Proposition. Let $X$ be a compact Boolean space, and let $\Gamma$ be a pointwise periodio group of homeomorphisms of $X$ with separated orbits. Then the set of orbital atoms is a basis for the topology of $X$. If $\Gamma$ is periodic, then the orders of the orbital atoms are bounded.

Proof. Let $x \in X$; then $x$ is a fixed point for the group $\Gamma_{x}$. By 3.7, there is a neighborhood basis of compact open sets invariant under $\Gamma_{x}$. Then, by 3.4, $x$ has a neighborhood basis of orbital atoms. The orders of orbital atoms are bounded if points have bounded order.

THEOREM II. If $\Gamma$ is a pointwise periodic group of homeomorphisms of a compact Boolean space $X$ with separated orbits, then the dual group $G=\Gamma^{*}$ is pointwise periodic on the dual Boolean algebra $A=X^{*}$.

Proof. Let $U$ be any compact open set; $U$ is the union of a finite collection $U_{1}, \ldots, U_{n}$ of orbital atoms (3.5). The number of translates $\gamma U_{i}$ of each is finite, and hence the total number of all the sets $\gamma_{1} U_{1} \cup \ldots \cup \gamma_{n} U_{n}, \gamma_{1}, \ldots, \gamma_{n} \in \Gamma$, is finite. Any translate of $U$ is one of these sets.

This result establishes statement (iii) of the introduction. We note that it has been proved only when $X$ is compact, or equivalently, if the Boolean ping has an identity (is a Boolean algebra), where Wallace [8] was able to prove this for a cyclic group on a locally compact Boolean space:

THEOREM III (Wallace). If $\Gamma$ is a cyclic pointwise periodic group of homeomorphisms of a locally compact Boolean space $X$, then its dual group $G=\Gamma^{*}$ is pointwise periodic on the dual Boolean ring $A=X^{*}$.

This theorem obviously remains true if "cyclie" is replaced by "finitely generated abelian".

We have also established somewhat more than Theorem III, in that we are able to give an analogous statement to (a) of the introduction. The following terminology is convenient:

3.9. Definition. Let $G$ be a pointwise periodic group of automorphisms of a Boolean ring $A$. Then $G$ is said to be atomically periodic if the orders of the orbital atoms are bounded.

THEOREM IV. Let $G$ be a group of automorphisms of a Boolean algebra A, let $X=A^{*}$, and let $\Gamma=G^{*}$ be the dual group of homeomorphisms. If $G$ is pointwise periodic and atomically periodic, then $\Gamma$ is periodic. If $\Gamma$ is periodic and has separated orbits, then $G$ is pointwise periodic and atomically periodic.

This follows at once from Proposition 3.1 and 3.8, and Theorem II.

The assumption that $T$ has separated orbits cannot be omitted in the above theorems, as the example $(5.4$, below) shows. More precisely, the following is true:

THEOREM V. Let $G$ be a group of automorphisms of a Boolean algebra $A$ and let $\Gamma$ be the dual group of homeomorphisms on the compact Boolean space $X=A^{*}$. If $T$ is pointwise periodic, then $G$ is pointwise periodic if and only if $\Gamma$ has separated orbits.

Proof. We need only show that if both $\Gamma$ and $G$ are pointwise periodic, then $\Gamma$ has separated orbits. Let $x, y \in X$ have disjoint orbits, and let $U$ and $U^{\prime}$ be disjoint neighborhoods of these orbits, respectively. Such neighborhoods exist because $X$ is normal. We will show the existence of an invariant compact open neighborhood $Z$ of $x$ which is contained in $U$; analogously, there will exist a similar neighborhood of $y$ in $U^{\prime}$. Projecting these neighborhoods into the orbit space $Y$ by the projection $\pi$ will yield disjoint neighborhoods of $\pi(x)$ and $\pi(y)$, which will prove the theorem.

Let $\left\{\gamma_{0} x, \gamma_{1} x, \ldots, \gamma_{n} x\right\}$ be the orbit of $x$, where $\gamma_{0}=1$. By 2.2 , we can select orbital atoms $V_{0}, \ldots, V_{n}$ such that $\gamma_{i} x \in V_{i} \subset U, i=0,1, \ldots, n$. 
If $W_{i}=\gamma_{i}^{-1} V_{i}$, then $W_{i}$ is an orbital atom and a neighborhood of $x$. Let $W=\bigcap_{i=0}^{n} W_{i}$; then $W$ is an orbital atom (2.3), and $x \in W$. If $\Gamma_{x}$ is the fix group, then $\Gamma_{x} W=W$, since $W$ is an orbital atom. Now let $Z=\Gamma W$. Then $Z$ is clearly invariant, and $T W=\left(\gamma_{0} \Gamma_{x} \cup \ldots \cup \gamma_{n} \Gamma_{x}\right) W=W \cup \gamma_{1} W \cup$ $\cup \ldots \cup \gamma_{n} W \subset U$. This accomplishes the proof.

4. $\sigma$-rings. Throughout this section, $A$ will be a Boolean $\sigma$-ring; that is, any countable subset of $A$ has a least upper bound and a greatest lower bound. The dual space $X=A^{*}$ of such a Boolean ring will be called a $\sigma$-space. These spaces are characterized by the property that the union of a countable collection of compact open sets has a compact open closure. Everything we say here applies, in particular, to complete Boolean algebras, or to extremally disconnected Boolean spaces (every open set has a compact open closure).

For this section, we shall always assume that $G$ is a pointwise periodic group of automorphisms of $A$. Our task is to prove that $G$ is then necessarily a finite group.

4.1. Propostrion. Let $E$ be a countable set of non-zero orbital atoms. If $b=\inf E$, then $b$ is an orbital atom, and if $b \neq 0$, then $o(b) \geqslant \sup \{o(a): a \in E\}$.

Proof. If $b \wedge b^{a} \neq 0$, then none of the elements $a_{1} \vee a^{g}$, where $a_{1}, a \in E$, can be 0 . In particular, $a \wedge a^{g} \neq 0$ which implies $a=a^{g}$, for each $a \in E$. In turn, $b=b^{g}$. Then $b=b^{g}$ implies $a=a^{g}$ for all $a \in E$, and hence $G_{b}\left(G_{a}\right.$. Therefore $o(b) \geqslant o(a)$ for each $a \in E$.

The notation introduced in the next definition is of considerable utility in the subsequent analysis.

4.2. Definimion. Two elements $a$ and $b$ are said to be separated if $a \wedge \bar{b}=0$. A subset $E$ is separated if each pair in $E$ is separated.

It follows at once from 2.5 that $a$ and $b$ are separated if and only if $\bar{a} \wedge \bar{b}=0$, so that this is a symmetric property.

4.3. Proposirion. Let $E$ be a countable separated set of non-zero elements of $A$, with. $b=\sup E$. Then $o(b) \geqslant \sup \{o(a): a \in E\}$.

Proof. Let $a \in E$, and suppose that $b=b^{g}$. Then $\bar{a} \wedge b=\bar{a} \wedge b^{g}$. Since $E$ is separated, then $\bar{a} \wedge b=a$, and, after transforming by $g, \bar{a} \wedge b^{g}=a^{g}$. This proves the proposition.

4.4. Propostiton. Let $\left\{a_{n}\right\}$ be a separated sequence of non-zero orbital atoms. Then the orders of the elements in the sequence are bounded.

Proof. Since $G$ is pointwise periodic, the order of $\sup a_{n}$ is finite; by 4.3 , this order is an upper bound.

4.5. Proposition. Let $\left\{a_{n}\right\}$ be a decreasing sequence of orbital atoms. Then the orders of the elements in the sequence are bounded.
Proof. Suppose that the orders of the $a_{n}$ are unbounded. Then there exists a subsequence $a_{n(i)}=b_{i}$ such that $o\left(b_{1}\right) \geqslant 3, o\left(b_{i+1}\right) \geqslant 3 \cdot o\left(b_{i}\right)$, $i=1,2, \ldots$ We shall define inductively a sequence $g_{2}, g_{3}, \ldots$ of group elements so that the element $c$ of $A$ defined by setting $c=b_{1} \vee b_{2}^{g_{2}} \vee b_{3}^{\sigma_{3}} \vee \ldots$ cannot possibly have finite order. This contradiction will prove the proposition.

Set $g_{1}=1$. Since $o\left(b_{1}\right) \geqslant 3$, we can choose group elements $g_{2}, g_{3}$ so that the elements

$$
b_{1}, b_{1}^{g_{2}}, b_{1}^{g_{\mathrm{s}}}
$$

are disjoint. Let $b_{0}=b_{1}^{g_{3} a_{2}^{-1}}$. By induction we can select a sequence $g_{1}=1$, $g_{2}, g_{3}, \ldots, g_{n}, \ldots$ so that

$$
\begin{gathered}
b_{j-1}^{g j+1}<b_{j-2}^{g g}, \\
b_{j-1}^{g j+1} \wedge b_{j-1}^{g j}=0,
\end{gathered}
$$

for $j=2,3, \ldots$ We have in fact done this for $j=2$; if we suppose that $g_{j}$ has been defined for $2 \leqslant j \leqslant n+1, n \geqslant 1$, we can choose $g_{n+2}$ because $\theta\left(b_{n}\right) \geqslant 3 \cdot o\left(b_{n-1}\right)$. A repeated application of (i) gives

$$
b_{n-1}^{a_{n+1}}<b_{k}^{g_{k+2}}, 1 \leqslant k \leqslant n-2 .
$$

Since

$$
b_{k}^{g_{k+2}} \wedge b_{k}^{g_{k+1}}=0
$$

by (ii) with $j=k+1$, we have

$$
b_{n-1}^{g_{n+1}} \wedge b_{k}^{g_{k+1}}=0, \quad 1 \leqslant k \leqslant n-2 .
$$

Since $b_{n+1}<b_{n-1}$, then

and therefore we have

$$
b_{n+1}^{g_{n+1}}<b_{n-1}^{g_{n+1}}
$$

$$
b_{n+1}^{g_{n+1}} \wedge b_{k}^{g_{k+1}}=0, \quad 1 \leqslant k \leqslant n-2 .
$$

By (ii) with $j=n$, we have $b_{n-1}^{g_{n+1}} \wedge b_{n-1}^{g_{n}}=0$, so that

Hence we have shown that

$$
b_{n+1}^{g_{n+1}} \wedge b_{n-1}^{g_{n}}=0 \text {. }
$$

$$
b_{n+1}^{\sigma_{n+1}} \wedge\left(b_{1}^{g_{2}} \vee b_{2}^{g_{3}} \vee \ldots \vee b_{n-1}^{g_{n}}\right)=0 \quad \text { for } \quad n \geqslant 1 .
$$

Since $b_{k+1}<b_{k}$, we have also shown that

$$
b_{n+1}^{g_{n+1}} \wedge\left(b_{2}^{g_{2}} \vee \ldots \vee b_{n}^{a_{n}}\right)=0, \quad n \geqslant 1 .
$$

Since $b_{n+1}^{g_{n+1}}<b_{n-1}^{\sigma_{n+1}}<b_{1}^{\sigma_{3}}$, and since $b_{1}=b_{1}^{g_{1}}$ is disjoint from $b_{1}^{\sigma_{3}}$, we also have $b_{n+1}^{g_{n+1}} \wedge b_{1}=0$. Thus the sequence

is disjoint.

$$
b_{1}, b_{2}^{g_{2}}, b_{3}^{\sigma_{3}}, \ldots
$$


Now set $c=b_{1} \vee b_{2}^{g_{2}} \vee b_{3}^{g_{3}} \vee \ldots$, and suppose that $e^{g}=c$ for some $g \in G$. If $b_{1}^{\sigma} \neq b_{1}$, then $b_{1} \wedge b_{1}^{g}=0$, since $b_{1}$ is an orbital atom. Therefore

$$
\begin{aligned}
b_{1}^{g} & =b_{1}^{g} \wedge c^{g}=b_{1}^{g} \wedge c=b_{1}^{g} \wedge\left(b_{1} \vee b_{2}^{g_{2}} \vee b_{3}^{g_{3}} \vee \ldots\right) \\
& =\left(b_{1}^{g} \wedge b_{2}^{g_{2}}\right) \vee\left(b_{1}^{g} \wedge b_{3}^{g_{3}}\right) \vee \ldots
\end{aligned}
$$

By (iii), we have $b_{k}^{g_{k+2}}<b_{1}^{g_{3}}, k \leqslant 1$. Since $b_{1}^{g_{3}} \wedge b_{1}^{g_{2}}=0$, we have $b_{1}^{g} \wedge b_{1}^{g_{2}}$ $=b_{1}^{g} \wedge b_{2}^{g_{2}} \wedge b_{1}^{g_{2}}=b_{1}^{g} \wedge b_{2}^{g_{2}}$. Unless this reduces to $0=0$ it asserts the absurd equality of two orbital atoms of different order. Hence we must have $b_{1}^{g} \wedge b_{2}^{g_{2}}=0$. A simple induction continues this argument, and shows that $b_{1}^{g} \wedge b_{n}^{g_{n}}=0$ for all $n$. Since this implies that $b_{1}^{g}=0$, which is impossible, we must conclude that $b_{1}^{\theta}=b_{1}$. In other words, $e^{\theta}=c$ implies $b_{1}^{o}=b_{1}$.

Suppose that we have shown that $c^{g}=c$ implies $b_{j}^{g j o}=b_{j}^{\eta j}, 1 \leqslant j \leqslant n$. If we set $d=b_{n+1}^{g_{n+1}} \vee b_{n+2}^{g_{n+2}} \vee \ldots$, then disjointness and $e^{g}=c$ imply $d^{0}=d$. If $b_{n+1}^{g_{n+1} g} \neq b_{n+1}^{g_{n+1}}$, then we can show, just as before, that $b_{n+1}^{a_{n+1} g} \wedge b_{n+1}^{g_{n+1}}=0$ and that $b_{n+1}^{g_{n+1} g} \leqslant b_{n+2}^{g_{n+2}} \vee b_{n+3}^{g_{n+3}} \vee \ldots$ Intersecting with $b_{n+1}^{g_{n+2}}$ and applying (iv), we obtain $b_{n+1}^{g_{n+1} g} \wedge b_{n+1}^{q_{n+2}}=b_{n+1}^{g_{n+1} g} \wedge b_{n+2}^{g_{n+2}}$. Again, this must reduce to $0=0$, so that $b_{n+1}^{g_{n+1} g} \wedge b_{n+2}^{g_{n+2}}=0$, and $b_{n+1}^{a_{n+1}} \leqslant b_{n+3}^{g_{n+3}} \vee b_{n+4}^{a_{n+4}} \vee \ldots$ Repeating the argument of the above paragraph, we have

$$
b_{n+1}^{g_{n+1} g}=b_{n+1}^{g_{n+1}} .
$$

Hence $c^{g}=o$ implies $b_{n}^{g_{n} g}=b_{n}^{g_{n}}, n=1,2, \ldots$ In other words, $G_{c}$ $\subset g_{n}^{-1} G_{b_{n}} g_{n}$ for all $n$. Therefore $o\left(b_{n}\right) \mid o(c)$ for all $n$. Since $o\left(b_{n}\right) \rightarrow \infty$ as $n \rightarrow \infty$, this contradicts the hypothesis that $o(c)$ is finite, and completes the proof.

4.6. Proposirron. There is no sequence of non-zero orbital atoms with unbounded orders.

Proof. It suffices to show that any such sequence $\left\{a_{n}\right\}$ contains a subsequence with bounded orders. We define a triangular matrix by setting

$$
\begin{aligned}
& a_{n n}=a_{n} \quad \text { for } \quad n=1,2, \ldots, \\
& a_{n m}=a_{m} \wedge \overline{a_{m+1}} \wedge \overline{a_{m+2}} \wedge \ldots \wedge \overline{a_{n}} \quad \text { for } \quad m<n .
\end{aligned}
$$

Then $a_{n m}$ is an orbital atom, and $\overline{a_{n m}}=\overline{a_{m}} \wedge \overline{a_{m+1}} \wedge \ldots \wedge \overline{a_{n}}$, by 2.3 and 2.5 . If $n \leqslant n^{\prime}$, and $m<m^{\prime}$, then $a_{n m} \wedge \overline{a_{n^{\prime} m^{\prime}}}=a_{n^{\prime} m}$; for $a_{n m} \wedge \overline{a_{n^{\prime} m^{\prime}}}=a_{m} \wedge \overline{a_{m+1}} \wedge$ $\wedge \ldots \wedge \overline{a_{n}} \wedge \overline{a_{m^{\prime}}} \wedge \ldots \wedge \overline{a_{n^{\prime}}}=a_{m} \wedge \overline{a_{m+1}} \ldots \wedge \overline{a_{n^{\prime}}}=a_{n^{\prime} m}$. The following are obvious from the definition:

$$
\begin{array}{lll}
m \leqslant n<n^{\prime} & \text { implies } & a_{n^{\prime} m} \leqslant a_{n m} . \\
m<m^{\prime} \leqslant n & \text { implies } & a_{n m} \leqslant a_{n m^{\prime}},
\end{array}
$$

By 2.3, $o\left(a_{n n}\right)=o\left(a_{n}\right)$ divides $o\left(a_{n m}\right)$ for all $m \leqslant n$, provided $a_{n m} \neq 0$. Therefore, if for at least one $m$, the decreasing sequence $\left\{a_{n m}: n=m\right.$, $m+1, \ldots\}$ is not finally zero, the sequence $\left\{a_{n n}\right\}=\left\{a_{n}\right\}$ is of bounded order, since $\left\{a_{n m}: n=m, m+1, \ldots\right\}$ is of bounded order, by lemma 4.5. Suppose now that all the columns are finally zero. Let $s(m)$ be the smallest row index such that $a_{n m}=0$ for all $n \geqslant s(m)$. We define by recursion two functions $f$ and $g$ fiom the natural numbers to the natural numbers:

$$
\begin{aligned}
& f(1)=g(1)=1 ; \\
& f(n)=s(g(n-1)), \quad g(n)=\min \left\{m^{\prime}: a_{f(n) m^{\prime}} \neq 0\right\} .
\end{aligned}
$$

Note that $g(n)$ is well-defined, since $a_{f(n) 1}=0$ and $a_{f(n) f(n)}=a_{f(n)} \neq 0$. It is clear from the definition that $f$ and $g$ are strictly increasing functions. We put $b_{n}=a_{f(n) g(n)}$. Then $\left\{b_{n}\right\}$ is a sequence of orbital atoms, and $o\left(a_{f(n) f(n)}\right)=o\left(a_{f(n)}\right)$ divides $o\left(b_{n}\right)$. But the sequence $\left\{b_{n}\right\}$ is in fact a separated sequence. For, suppose $n<n^{\prime}$, then $b_{n} \wedge \overline{b_{n^{\prime}}}=a_{f(n) g(n)} \wedge \overline{a_{f\left(n^{\prime}\right) g\left(n^{\prime}\right)}}=$ $=a_{f\left(n^{\prime}\right) g(n)}$ by an observation atove. But $f\left(n^{\prime}\right) \geqslant f(n+1)=s(g(n))$, so that $a_{f\left(n^{\prime}\right) g(n)}=0$. Separated sequences of orbital atoms have, however, bounded orders, by 4.4. Hence $\left\{a_{f(n)}\right\}$ is a subsequence of the $\left\{a_{n}\right\}$ of bounded order. This accomplishes the proof.

4.7. Proposition. Let $G$ be a group of automorphisms of a Boolean $\sigma-r i n g$. Then, if $G$ is pointwise periodic, $G$ is atomically periodic.

This is another way of expressing the previous result. At this point, we may pause to take stock of the situation. For a cyclic group $G$, our proof is complete. For we may take the subring $A_{n}$ of $A$ consisting of all elements having an order which divides $n$ ! This is an increasing sequence of subrings. It is is not eventually constant, then there exist elements of increasing order, and hence there exist orbital atoms of increasing order, which contradicts 4.6. For the non-cyclic case (not to mention the non-abelian case), it is not clear that there exist orbital atoms of increasing order. Secondly, we note that 3.1 and 4.7 together immediately imply the following result:

4.9. Proposition. If $G$ is a pointwise periodic group of automorphisms of a Boolean $\sigma$-ring, then the dual group $\Gamma=G^{*}$ is periodic on $X=A^{*}$.

4.9. LenNa. Let $\left\{a_{i}\right\}$ be a separated sequence of orbital atoms. Let $G_{i}=G_{a_{i}}$ be the group of all elements leaving $a_{i}$ fixed. Then the collection $\left\{G_{i}\right\}$ of groups is finite.

Proof. Let $a=\sup a_{i}$. Then $a^{g}=a$ if and only if $a_{i}^{g}=a_{i}$, since the $a_{i}$ are separated. (Compare the proof of 4.3.) Hence $G_{a}=\bigcap_{i=1}^{\infty} G_{i}$. Let $H_{i}=G_{1} \cap G_{2} \cap \ldots \cap G_{i}$. Since all of the groups $G_{i}$ have finite index $\left(G: G_{i}\right)=o\left(a_{i}\right)$, the groups $H_{i}$ have finite index (Poincaré; see [4], p. 62). The intersection $G_{a}=\bigcap_{i=0}^{\infty} H_{i}$ has finite index $\left(G: G_{a}\right)=o(a)$. Therefore there is an integer $j$ such that $H_{j}=H_{j+1}=\ldots$ Thus $G_{a}=G_{i} \cap \ldots \cap G_{j}$, and the coset space $G / G_{a}$ has only a finite number of elements. If there Fundamenta Mathematicae, T. LII 
were infinitely many different groups $G_{i(n)}$ in the collection $\left\{G_{i}\right\}$, then there would have to be infinitely many coset spaces $G_{i(n)} / G_{a}$, which is impossible.

The center of action now shifts to the space $X$.

4.10. Lemra. Let $X$ be a locally compact Boolean space, and let $\Gamma$ be a pointwise periodic group of homeomorphisms of $X$ such that the orbital atoms form a basis for the topology of $X$. Then, for each point $x \in X$, there is a neighborhood $U$ of $x$ such that $y \in U$ implies $\Gamma_{y} \subset \Gamma_{x}$.

Proof. Let $U$ be an orbital atom which is a neighborhood of $x$ such that $\gamma x \neq x$ implies $\gamma x \notin U$; such a set $U$ exists because the orbital atoms form a base, and because points have finite orbit. Let $y \in U$; if $\gamma y=y$, then $\gamma^{-1} U \cap U \neq \varnothing$. Since $U$ is an orbital atom, $U=\gamma^{-1} U$. This implies $\gamma x \in U$, and by the choice of $U$, this implies $\gamma x=x$.

4.11. Definimion. Let $X$ be any topological space, and let $\Gamma$ be any group of homeomorphisms of $X$. Define, for $x, y \in X$, the relation $x \leqslant y$ to mean there exists an element $\gamma \in \Gamma$ such that $\Gamma_{x} C \gamma^{-1} \Gamma_{y} \gamma$.

4.12. Lemara. The relation $\leqslant$ defined in 4.11 is a partial order on $X$, and $x \leqslant y$ implies $\gamma x \leqslant \gamma y$ for any $\gamma \in \Gamma$.

The obvious proof of this may be omitted.

4.13. Lemara. Let $X$ be a locally compact Boolean space, and let $\Gamma$ be a pointwise periodio group of homeomorphisms of $X$ such that the orbital atoms form a basis for the topology of $X$. For any $x \in X$, the set $\{y \in X$ : $y \leqslant x\}$ is open.

Proof. Let $y \leqslant x$; there is an element $\gamma \in \Gamma$ such that $\Gamma_{\left(\gamma^{-1} y\right)} \subset \Gamma_{x}$. By 4.10, there is a neighborhood $U$ of $y$ such that $z \in U$ implies $\Gamma_{z} \subset \Gamma_{y}$. Then $z \in U$ implies $\Gamma_{\left(y^{-1} z\right)} \subset \Gamma_{x}$. This finishes the proof.

4.14. Lencara. Let $\Gamma$ be a periodic group of homeomorphisms of a locally compact Boolean space, such that the orbital atoms form a basis for the topology of the space. Then there exist minimal points in the partial order of 4.11, and the set $M$ of all minimal points is open, and invariant under $\Gamma$.

Proof. Since $T$ is periodic, there is a natural number $q$ such that the order $\left(\Gamma: \Gamma_{x}\right)$ of any point $x$ divides $q$; hence there are minimal groups in the collection of all $\left\{\Gamma_{x}: x \in X\right\}$. The set $M$ of all points $x$, whose group $\Gamma_{x}$ is minimal, is the set of all minimal points. By $4.13, M$ is open, and by $4.12, M$ is invariant under $\Gamma$.

4.15. LEMMa. Let $T$ be a periodic group of homeomorphisms of a locally compact Boolean space $X$, such that the orbital atoms form a basis for the topology of $X$. Let $\left\{\Gamma_{i}: i \in I\right\}$ be a collection of minimal groups in the family $\left\{\Gamma_{x}: x \in X\right\}$, such that each class of conjugates in the family $\left\{\Gamma_{x}: x \in M\right.$ contains precisely one member of the class $\left\{\Gamma_{i}: i \in I\right\}$. For each $i \in I$, let
$M_{i}=\left\{x \in M: \Gamma_{x}=\gamma^{-1} \Gamma_{i} \gamma\right.$ for some $\left.\gamma \in \Gamma\right\}$. Then, for each $i \in I$, the set $M_{i}$ is open and closed in $M$, is invariant under $\Gamma$, and $M=\bigcup_{i \in I} M_{i}$.

Proof. The family $\left\{M_{i}: i \in I\right\}$ is clearly a decomposition of $M$ into pairwise disjoint sets, each invariant under $\Gamma$; to prove the lemma it is therefore sufficient to show that each set $M_{i}$ is open. Let $x \in M_{i}$, then $\Gamma_{x}=\gamma^{-1} \Gamma_{i} \gamma$, for some $\gamma \in \Gamma$. By 4.10, there is a neighborhood $U$ of $x$ such that $y \in U$ implies $\Gamma_{y} \subset \Gamma_{x}=\gamma^{-1} \Gamma_{i} \gamma$. By the minimality of the groups $\Gamma_{i}$, these must be equal.

4.16. Lemara. Let $A$ be a Boolean $\sigma$-ring, let $G$ be a pointwise periodic group of automorphisms of $A$, let $X=A^{*}$ and $\Gamma=G^{*}$. Then the family $\left\{M_{i}: i \in I\right\}$ of 4.15 is finite.

Proof. By 2.2, the orbital atoms form a basis for the topology of $X$. By 4.8, $\Gamma$ is periodic on $X$. Assume, by way of contradiction, that there is a sequence $\Gamma_{i(n)}$ of different groups in $\left\{\Gamma_{i}: i \in I\right\}$. The set $M_{i(n)}$ is open; let $x_{n} \in M_{i(n)}$ such that $\Gamma_{x_{n}}=\Gamma_{i(n)}$. Then there exists an orbital atom $U_{n}$ which is a neighborhood of $x_{n}$ and which is contained in $M_{i(n)}$, and which has the property that $\gamma x_{n} \neq x_{n}$ implies $\gamma x_{n} \notin U_{n}$. The invariance of the sets $M_{i(n)}$ shows that the family $\left\{U_{n}\right\}$ is a separate sequence of orbital atoms, such that their fixed groups form an infinite family of different groups. This contradicts 4.9. The proof is complete.

4.17. Lemora. Let $A$ be a Boolean $\sigma$-ring, let $G$ be a pointwise periodic group of automorphisms of $A$, let $X=A^{*}$ and $\Gamma=G^{*}$. Then the collection $\left\{\Gamma_{x}: x \in X\right\}$ is finite.

Proof. By 4.16, $\left\{M_{i}: i \in I\right\}$ is a finite family, and $M$ is their union.

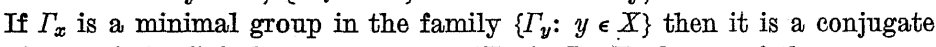
of one of the finitely many groups $\left\{\Gamma_{i}: i \in I\right\}$. Each one of these groups has only finitely many conjugates. Hence the collection of minimal groups in $\left\{\Gamma_{x}: x \in X\right\}$ is finite. Every group in this collection contains a minimal one, and the number of groups containing a given minimal group in the collection is finite, since the minimal groups have finite index. Consequently the entire collection must be finite.

4.18. Proposition. Let $X$ be a locally compact Boolean $\sigma$-space, let $A=X^{*}$, let $\Gamma$ be a group of homeomorphisms of $X$, and let $G=\Gamma^{*}$. If $G$ is pointwise periodic on $A$, then $\Gamma$ is a finite group.

Proof. By 2.2, the orbital atoms form a basis for the topology of $X$, and by 4.8 , the group $\Gamma$ is periodic on $X$. The intersection $\bigcap\left\{\Gamma_{x}: x \in X\right\}$ of all the fixed groups of points in $X$ is a finite intersection, by 4.17 , and therefore has finite index in $\Gamma([4]$, p. 62). On the other hand, this intersection is the group of all elements that leave every point in $X$ fixed. Only the identity homeomorphism does this (i.e., any homeomorphism 
group is effective). Thus $\Gamma$ has finite index over the identity subgroup, and is therefore finite.

We have therefore completed the proof of the following result, which is the principal theorem of this paper:

THEOREM VI. Let A be a Boolean $\sigma$-ring, and let $G$ be a group of automorphisms of $A$. Then the following statements are mutually equivalent:

(i) $G$ is a finite group;

(ii) $G$ is a periodic group on $A$;

(iii) $G$ is a pointwise periodic group on $A$.

COROLLARY VI.1. A pointwise periodic group of homeomorphisms of a locally compact Boolean $\sigma$-space is a torsion group.

Proof. Each element of such a group generates a cyclic subgroup, which induces a cyclic pointwise periodic automorphism group (Theorem II), which is, in turn, finite (Theorem VI).

CoRollary VI.2. Let $A$ be a Boolean o-ring, $X$ a locally compact Boolean $\sigma$-space, $G$ a finitely generated, solvable group of automorphisms of $A$, and $\Gamma$ a group of homeomorphisms of $X$. If $A=X^{*}$ and $G=\Gamma^{*}$, then the following statements are equivalent:

(i) $G$ is a finite group;

(ii) $\Gamma$ is a finite group;

(iii) $G$ is periodic on $A$;

(iv) $\Gamma$ is periodic on $X$;

(v) $G$ is pointwise periodic on $A$;

(vi) $\Gamma$ is pointwise periodic on $X$.

Proof. It is sufficient to show that (vi) implies (i). By Corollary VI.1, $G$ is a torsion group. We prove that a finitely generated solvable torsion group is finite. Let $G, G^{1}, \ldots, G^{(k)}, G^{(k+1)}=\{1\}$ be the sequence of successive commutator groups of $G$. We prove our assertion by induction on $k$. The group $G / G^{(k)}$ is a homomorphic image of $G$, hence is a finitely generated solvable torsion group. The $k$ th commutator subgroup of $G / G^{(k)}$ is trivial. By inductive hypothesis, $G / G^{(k)}$ is a finite group. Now, by Schreier's theorem ([4], Par. 36, II), the subgroup $G^{(k)}$, being of finite index, is finitely generated. But $G^{(k)}$ is abelian and, being a finitely generated torsion group, is therefore finite. Since both $G / G^{(k)}$ and $G^{\left(k_{k}\right)}$ are finite, $G$ is finite. This completes the proof. (The authors are indebted to B. Huppert for this proof.)

In the case the $\sigma$-ring has an identity (is a $\sigma$-algebra), we have proved the following:
THeOREM VII. Let $A$ be a Boolean $\sigma$-algebra, $X$ a compact Boolean $\sigma$-space, $G$ a group of automorphisms of $A$, and $\Gamma$ a group of homeomorphisms of $X$. If $A=X^{*}$ and $G=\Gamma^{*}$, then the following statements are mutually equivalent:

(i) $G$ is a finite group;

(ii) $\Gamma$ is finite group;

(iii) $G$ is periodic on $A$;

(iv) $T$ is periodic on $X$ and has separated orbits;

( $\nabla) G$ is pointwise periodic on $A$;

(vi) $\Gamma$ is pointwise periodic on $X$ and has separated orbits.

This follows at once from an application of Theorem II.

5. Examples and counter-examples. This section is devoted to showing why the assumption that $A$ is a $\sigma$-ring is needed to prove the results of section 4, and to showing that the converses of Theorems I and II do not hold. We also obtain one or two interesting consequences of the results of section 4 .

5.1. That a homeomorphism of a compact Boolean space can be pointwise periodic without being periodic has long been known; we include an example only for completeness. Let Pascal's triangle be regarded as a discrete space, and let $X$ be the one-point compactification. Let $\gamma$ be the transformation which maps the symbol $\left(\begin{array}{l}n \\ j\end{array}\right)$ onto the symbol $\left(\begin{array}{c}n \\ j+1\end{array}\right)$ for $j<n$, which maps $\left(\begin{array}{l}n \\ n\end{array}\right)$ onto $\left(\begin{array}{l}n \\ 0\end{array}\right)$, and which leaves $\infty$ fixed. Then $\gamma$ is pointwise periodic, but not periodic.

5.2. The dual algebra $A$ of the space $X$ in 5.1 is the algebra of all finite subsets and their complements in the discrete triangle; the dual of $\gamma$ is a pointwise periodic automorphism of $A$ which is not periodic.

5.3. If the space $X$ is taken to be the Stone-Cech compactification of the triangle, rather than the one-point compactification, the transformation $\gamma$ has an extension to $X$ which must, by section 4, have points of infinite order, since $X$ is now extremelly disconnected.

5.4. Let $X$ be the space of all integers compactified by adding $-\infty$ and $\infty$. The permutation $g_{i}=(-i, i)$ which interchanges $-i$ and $i$, and leaves all other elements fixed, is a homeomorphism, for all $i=1,2, \ldots$ The subgroup $\Gamma$ of the full automorphism group generated by $g_{1}, g_{2}, \ldots$ is an abelian group isomorphic to the vector space with countably infinite dimension over the field of two elements. All orbits contain either one or two elements, so that $\Gamma$ is periodic. The group $\Gamma^{*}=G$ on the ring of compact open sets is not even pointwise periodic. Let $V$ be the interval $[-\infty,-1]$, which is a compact open neighborhood of $-\infty$. For any two 
integers $i, j$ such that $i, j \geqslant 1, i \neq j$, the transforms $g_{i} \nabla$ and $g_{j} V$ are different.

The two points $-\infty$ and $\infty$ have no small invariant neighborhoods. The orbit space $Y$ of $X$ under $\Gamma$ is not a Hausdorff space because the images of $-\infty$ and $\infty$ in $Y$ cannot be separated. All orbital atoms are single points, and all have order one or two.

This example serves many purposes. It shows how drastically the converse of Theorem I fails. It shows that the assumption of separated orbits in Theorem II is not redundant, and that Theorem $V$ is not vacuous. It also shows that Lemma 3.7 fails without the assumption of separated orbits.

If the points $-\infty$ and $\infty$ are identified at the outset, another example of some utility is obtained. We now have an orbit space which is Hausdorff. The group $\Gamma$ is periodic on $X$, and the dual group $G=\Gamma^{*}$ is now pointwise periodic on $A=X^{*}$. Moreover, all orbital atoms have order $\leqslant 2$. However, it is clear that $G$ is not periodic on $A$, since the set $\{-n, \ldots, 0, \ldots, n\}$ has order $2^{n}$. This shows that the failure of the converse of Theorem I does not lie in the lack of separation of orbits.

5.5. Let $\Delta_{i}$ be the $i$-dimensional vector group over the field of two elements. Let $X$ be the one-point compactification of the disjoint union of the $\Delta_{i}$. Let $\Gamma$ be the restricted direct sum $\Delta_{1}+\Delta_{2}+\ldots$ acting on $X$ in such a fashion that $\Delta_{i}$ leaves all points fixed except the points of $\Delta_{i}$, where it acts by translation. Then $\Gamma$ is pointwise periodic but not periodic, since the order or the identity in $\Delta_{i}$ is $2^{i}$. The example is an analogue of 5.1, where the group is now a countable abelian torsion group of exponent 2.

5.6. Let $X_{i}$ be a set consisting of $2 i$ different points $a_{i 1}, \ldots, a_{i i}$, $b_{i 1}, \ldots, b_{i i}$, and let $X$ be the disjoint union of the $X_{i}, i=1,2, \ldots$ Let $\Gamma$ be the group generated by the following two permutations of $\bar{X}$ :

$$
\begin{aligned}
& \gamma_{1}=\left(a_{11} b_{11}\right)\left(a_{21} b_{21}\right)\left(a_{31} b_{31}\right) \ldots, \\
& \gamma_{2}=\left(a_{11}\right)\left(b_{11}\right)\left(a_{21} a_{22}\right)\left(b_{21} b_{22}\right)\left(a_{31} a_{32} a_{33}\right)\left(b_{31} b_{32} b_{33}\right) \ldots
\end{aligned}
$$

The orbits are the sets $X_{i}$, and hence $T$ is pointwise periodic but not periodic. This is a 2-step metabelian group, and is, in particular, solvable. ( $\Gamma$ is, in fact, the extension of a vector group of countably infinite dimension over the field $G F^{\prime}(2)$ by an infinite cyclic group.) This example shows that the hypothesis that $X$ is a $\sigma$-space in Corollary VI.2 is essential. If $X$ is compactified by adding a single point invariant under $\Gamma$, one sees that compactness has nothing to do with this. (The authors are indebted to $H$. Wielandt for this example.)

5.7. Proposimion. Let $X$ be a totally disconnected compact topological group, and let $\left\{U_{i}: i \in I\right\}$ be a basis for neighborhoods of the identity con- sisting of compact open subgroups. Then the Boolean algebra $A$ of compact open subsets of $X$ is generated by the sets $\left\{U_{i} x: i \in I, x \in X\right\}$, and any subgroup $\Gamma$ of $X$ induces a pointwise periodic group of automorphisms of $A$.

Proof. Let $U$ be any compact open set in $X$. Then for each $x \in U$, there is a neighborhood $U_{i(x)}, i(x) \in I$, such that $U_{i(x)} x$ is contained in $U$. Since $U$ is compact and is covered by these translates, there is a finite set of points $x_{1}, \ldots, x_{n}$ such that $U=U_{i\left(x_{1}\right)} x_{1} \cup \ldots \cup U_{i\left(x_{n}\right)} x_{n}$. Thus $A$ is generated by the indicated sets. Let $\Gamma$ be any subgroup of $X$, acting by right translation. Since all the sets $U_{i}$ are subgroups, they are orbital atoms in $A$ under $G=\Gamma^{*}$. Since they are open subgroups, they have finite index. Hence all the $U_{i}$ have finite orbits, which implies that every compact open set is a union of finite collection of translates of the $U_{i}$.

CoRoLLARY VI.3. The space of a compact totally disconnected group is a $\sigma$-space if and only if the group is finite.

Since every locally compact totally disconnected group contains a compact open subgroup, and since a compact open subspace of a $\sigma$-space is a $\sigma$-space, it follows that if the space of a locally compact totally disconnected group is a $\sigma$-space, then the group is discrete, hence finite.

CoRollari VI.4. A compact $\sigma$-space is a product space of a collection of finite sets if and only if it is finite.

Proof. All such products admit a group structure.

These two corollaries apply in particular to extremally disconnected spaces.

5.8. Let $X$ be the group of all $p$-adic integers and $\Gamma$ the cyclic subgroup generated by the integer 1 . Then all orbits of $\Gamma$ are infinite, but the group induced on $A=X^{*}$ is pointwise periodic, by 5.6. This shows that the converse of (c) in the introduction does not hold in general.

5.9. Let $X$ be an infinite product of the groups with two elements, and endow $X$ with the Tychonoff topology. Let $X=\Gamma$. Then all orbits in $X$ are infinite, although $G=T^{*}$ is pointwise periodic, by 5.6. Thus the converse of (iii) in the introduction does not hold, even for a countable abelian torsion group of exponent 2 .

6. Separated orbits. The problem of determining when a group of homeomorphisms has separated orbits is part of a more general problem in the theory of transformation groups. In this section, we give a very brief account of some conditions sufficient for this. Details and references may be found in [1]; we follow the terminology of this reference.

Let $\Gamma$ be an effective topological transformation group acting on the topological space $X$. For each $x \in X$, let $\Gamma x$ denote the orbit of $x$, and let $\overline{\Gamma x}$ denote the closure of the orbit of $x$. Assume that the collection 
$\{\overline{\Gamma x}: x \in X\}$ is a partition of $X$. If $\bar{Y}$ denotes the quotient space of $X$ by the relation, and if $\pi$ denotes the projection of $X$ onto $Y$, then $\pi$ is a continuous open mapping ( $[1]$, p. 16, Remark 2.30), and $Y$ is a $T_{1}$-space.

Consider now this general setting: Suppose that $X$ is a topological space, $Y$ is a $T_{1}$-space which is the quotient of $X$ by some equivalence relation (with closed equivalence classes), and $\pi$ is the projection of $X$ onto $Y$. If $X$ is a locally compact Hausdorff space, if $\pi$ is a closed mapping and if the equivalence classes are compact, then $Y$ is a locally compact Hausdorff space ([3], p. 148). If $\pi$ is also open and $X$ is a Boolean space, then $Y$ is also a Boolean space.

The case which interests us here falls under these conditions. If $\Gamma$ is a pointwise periodic group of homeomorphisms of locally compact totally disconnected Hausdorff space $X$, where $T$ has the discrete topology, then the orbits are finite, a fortiori compact, and the projection is open. We are therefore interested in determining when the projection is a closed mapping.

This has a solution in the more general setting. For the purpose of formulating the solution, we introduce the following terminology, following [1], p. 32, Remark 4.12 (H).

6.1. Definition. Let $\Gamma$ be a topological group acting effectively on a topological space $X$. The group $\Gamma$ is said to be locally weakly almost periodic if, for each point $x \in X$ and each neighborhood $U$ of $x$, there exists a neighborhood $\nabla$ of $x$ and a compact subset $\Delta$ of $\Gamma$ such that $\Gamma V \subset \Delta U$.

For locally compact Hausdorff spaces, there is the following characterization of this notion ([1], p. 33, Theorem 4.17), which we reformulate in the language of this paper.

6.2. Proposition. Let $X$ be a locally compact Hausdorff space, and let $\Gamma$ be a topological group acting effectively on $X$. Then the following statements are pairwise equivalent:

(i) $\Gamma$ is locally weakly almost periodic on $X$;

(ii) for any point $x \in X$ and any neighborhood $U$ of $x$, there exists a neighborhood $\nabla$ of $x$ such that the orbit of $V$ under $\Gamma$ can be covered by a finite number of translates of $U$;

(iii) the collection of sets $\{\overline{\Gamma x}: x \in \bar{X}\}$ forms a partition of $X$ having compact equivalence classes, such that the projection of $X$ onto the orbit space $\bar{Y}$ is a closed mapping.

Now, let us suppose that $\Gamma$ is a pointwise periodic group of homeomorphisms of a compact Hausdorff space $X$. If $\Gamma$ has separated orbits, then the orbit space $Y$ is a compact Hausdorff space and the projection $\pi$ of $X$ onto $Y$ is a closed mapping. Conversely, if $\pi$ is a closed mapping, then, since orbits (equivalence classes) are compact, the earlier remarks of this section show that the orbit space $Y$ is a compact Hausdorff space. In other words, if $\Gamma$ is a pointwise periodic group of homeomorphisms of $X$, then a necessary and sufficient condition that $\Gamma$ have separated orbits is that the projection $\pi$ be a closed mapping. This, together with 6.2 , gives the following:

6.3. Proposition. Let $\Gamma$ be a pointwise periodic group of homeomorphisms of a compact Hausdorff space $X$. Then $\Gamma$ has separated orbits if and only if $\Gamma$ is locally weakly almost periodic.

In view of this, we can rephrase our principal results as follows:

THEOREM $\mathrm{II}^{\prime}$. If $\Gamma$ is a pointwise periodic and locally weakly almost periodic group of homeomorphisms of a compact Boolean space $X$, then the dual group $G=\Gamma^{*}$ is pointwise periodic on the dual Boolean algebra $A=X^{*}$.

THEOREM IV'. Let $G$ be a group of automorphisms of a Boolean algebra $A$, let $X=A^{*}$, and let $\Gamma=G^{*}$ be the dual group of homeomorphisms. Then $G$ is pointwise periodic and atomically periodic if and only if $\Gamma$ is periodic and locally weakly almost periodic.

THEOREM $V^{\prime}$. Let $G$ be a group of automorphisms of a Boolean algebra $A$, and let $\Gamma$ be the dual group of homeomorphisms on the compact Boolean space $X=A^{*}$. If $\Gamma$ is pointwise periodic, then $G$ is pointwise periodic if and only if $\Gamma$ is locally weakly almost periodic.

THEOREM VII'. Let $A$ be a Boolean $\sigma$-algebra, $X$ a compact Boolean $\sigma$-space, $G$ a group of automorphisms of $A$, and $\Gamma$ a group of homeomorphisms of $X$. If $A=X^{*}$ and $G=\Gamma^{*}$, then the following are mutually equivalent:

(i) $G$ is finite group,

(ii) $\Gamma$ is a finite group,

(iii) $G$ is periodic on $A$,

(iv) $\Gamma$ is periodic and locally weakly almost periodic on $X$,

( $\nabla) G$ is pointwise periodic on $A$,

(vi) $\Gamma$ is pointwise periodic and locally weakly almost periodic on $X$.

\section{References}

[I] W. H. Gottschalk and G. A. Hedlund, Topological dynamics, Amer. Math. Soc. Colloquium Publications, vol. 36, New York 1955.

[2] D. W. Hall and G. E. Schweigert, Properties of invariant sets under pointwise periodic homeomorphisms, Duke Math. J. 4 (1938), pp. 719-724.

[3] J. L. Kelley, General topology, New York 1955.

[4] A. Kurosch, Theory of groups, New York 1955

[5] D. Montgomery, Pointwise periodic homeomorphisms, Amer. J. Math. 59 (1937), pp. 118-120. 
[6] M. H. Stone, The theory of representations for Boolean algebras, Trans. Amer. Math. Soc. 40 (1936), pp. 37-111.

[7] A. D. Wallace, A local property of pointwise periodic homeomorphisms, Colloquium Mathematicum 9 (1962), pp. 63-66.

[8] F. B. Wright, Reourrence theorems and operators on Boolean algebras, Proc. London Math. Soc. (3) 11 (1961), pp. 385-401.

UNIVERSITXT TUBINGEN

TULANE UNIVERSITY OF LOUISIANA

Reģu par la Rédaction le 2\%.11.1961

\section{Perfect transfinite numbers}

by

\section{P. Zvengrowski (Chicago, 111.)}

1. Introduction. The well-known concept of a perfect (finite) number can easily be extended to include transifinite ordinal numbers provided some care is taken to take account of the non-commutativity of addition and multiplication of ordinals. This is done as follows.

1.1. Definimion. An ordinal number is left perfect if and only if it is equal to the sum of its proper left hand divisors, arranged in increasing order.

Right perfect is similarly defined. As an example, $\omega$ has the proper left hand divisors $1,2,3, \ldots$, and $1+2+3+\ldots=\omega$, so $\omega$ is left perfect. For finite ordinals, it is clear that the concepts of left perfect, right perfect, and the usual concept of perfect coincide.

In this paper it is proved that there are no transfinite right perfect ordinals, and all transfinite left perfect ordinals are determined to within certain questions of finite arithmetic (see Section 4).

2. Triangular numbers. In this section we develop certain properties of triangular numbers which will be needed later. All variables $\alpha, \delta, \sigma, \ldots$ will denote ordinals.

2.1. Definition. $T(\alpha)=\sum_{\zeta<a} \zeta$, all summations being understood to be taken in increasing order.

$T(\alpha)$ is simply the triangular number associated with $\alpha$. The function $T$ is obviously increasing and continuous. We wish to determine $T(\varrho)$ explicitly whenerer $\varrho$ is a prime component of addition.

2.2. Lenara. Let $\varrho$ be a prime component of addition, $\zeta_{\varphi}$ be a nondecreasing set of ordinals defined for $\varphi<\varrho$. Then for any $\beta<\varrho$ we have $\sum_{\beta \leqslant 4<\varrho} \zeta_{\varphi}=\sum_{\varphi<Q} \zeta_{\varphi}$.

Proof. $\beta<\varrho$ implies $\varrho=\beta+\varrho$. The $\zeta_{\varphi}$ are non-decreasing, hence $\sum_{\gamma<\beta} \zeta_{\phi} \leqslant \zeta_{\beta} \beta$.

Similarly, $\begin{array}{r}\sum_{\beta \leqslant q<\varrho} \zeta_{\varphi} \geqslant \zeta_{\beta}(\varrho-\beta)=\zeta_{\beta} \varrho, \text { say } \\ \sum \zeta_{\varphi}=\zeta_{\alpha} \varrho+\delta .\end{array}$

$$
\sum_{\beta \leqslant \psi<\varrho} \zeta_{\varphi}=\zeta_{\alpha} \varrho+\delta
$$

